Helgoländer wiss. Meeresunters. 17, 64-73 (1968)

\title{
Ermittlung von Bewegungsvorgängen im Meere und in Flußmündungen zur Untersuchung des Transportes von Verunreinigungen
}

\author{
HANS-Gerhard RAMming \\ Institut für Meereskunde der Universität Hamburg, Hamburg
}

\begin{abstract}
Investigation of motion processes in the sea and in estuaries for the study of the transport of pollutants. The hydrodynamical-numerical method developed by HANSEN (1967) makes it possible to determine the motion in oceanic areas, in adjacent and marginal seas, in shelf areas, as well as in estuaries and rivers. In many cases the method has been successfully used in reproducing tides and storm surges quantitatively. For the numerical treatment of the hydrodynamical equations of motion the natural conditions, such as depth distribution, boundary values and - as far as these are effective - meteorological data and density distribution were considered. In shallow water areas the tides are a combination of harmonic and nonharmonic constituents; the nonlinear parts cause in a horizontal direction a residual current and in a vertical direction a deviation from the mean sea level. In this way it becomes possible to investigate the distribution of water pollution in tidal rivers and in the North Sea.
\end{abstract}

\section{EINLEITUNG}

Das von HANSEN $(1966,1967)$ entwickelte hydrodynamisch-numerische Verfahren ermöglicht die Ermittlung der Bewegungsvorgänge in Ozeanen, in Rand- und Nebenmeeren und deren Küstenbereich sowie in den Flußmündungen und Flüssen. Das Verfahren ist in vielen Fällen zur quantitativen Reproduktion von Gezeiten und Sturmfluten mit Erfolg verwendet worden. Bei der numerischen Behandlung der hydrodynamischen Bewegungsgleichungen werden die natürlichen Gegebenheiten, wie die Tiefenverteilung, gemessene Randwerte und, soweit wirksam, meteorologische Daten und die Dichteverteilung berücksichtigt. Die Bewegungsvorgänge im Meere und in Gezeitenflüssen sind im wesentlichen bedingt durch Gezeiten, Wind und Dichteunterschiede sowie durch Oberwasserzufluß. Diese vertikalen und horizontalen Wasserbewegungen werden während ein es Prozesses unter voller Berücksichtigung der Wechselwirkung gleichzeitig ermittelt. Im wesentlichen soll hier über die Ermittlung der Bewegungsvorgänge in Gezeitenflüssen berichtet werden. 


\section{ERGEBNISSE UND DISKUSSION}

Sind an der Mündung eines Gezeitenflusses der Verlauf des Wasserstandes in Abhängigkeit von der Zeit, oberhalb der Gezeitengrenze die Geschwindigkeit bzw. die Oberwassermenge und innerhalb des Flusses die Tiefen- und Breitenverteilung bekannt, dann lassen sich im Innern des Flusses Wasserstände und Geschwindigkeiten berechnen.
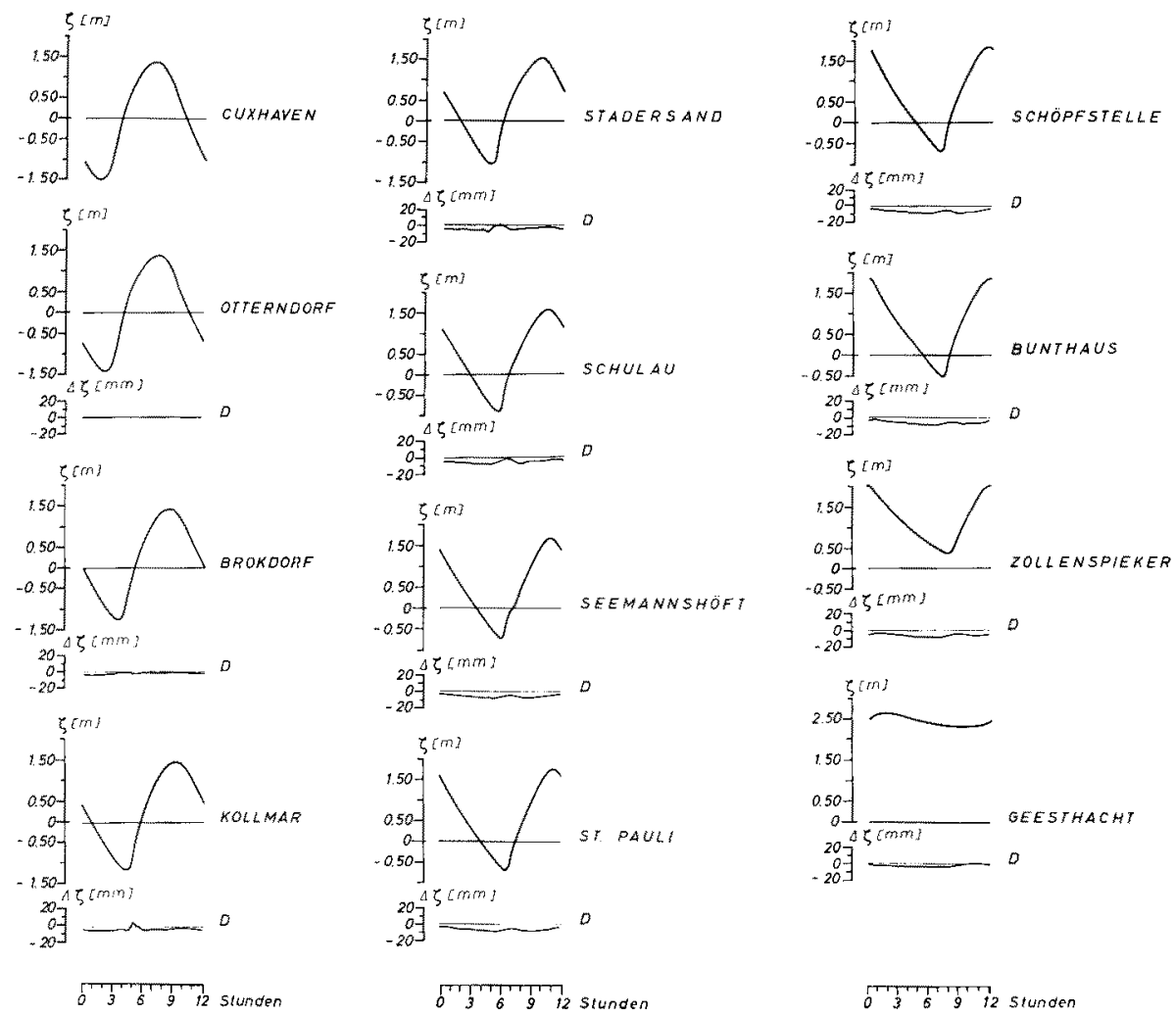

$D=$ Ditferenz zwischen Messung und Rechnurg in Miltmetern

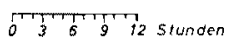

Abb. 1: Die Elbe, Normaltide 1951/1955, Messung und Rechnung

Hierzu wird die Flußstrecke in Streckenabschnitte unterteilt. Zwischen zwei Wasserstandsrechenpunkten liegt jeweils ein Geschwindigkeitsrechenpunkt. Um die Feinstruktur der Tiefenverteilung und der Querschnittsformen zu erfassen, werden die Querschnitte durch Parallelkanäle verschiedener Breiten und Tiefen approximiert. In Kanälen mit größerer Tiefe und damit geringerer Reibung sind die Geschwindigkeiten und die Transporte im Mittel größer und in Kanälen mit geringerer Tiefe und größerer Reibung entsprechend kleiner. Nach der Berechnung der Geschwindigkeiten eines Zeitschrittes in jedem Kanal kann die Durchflußmenge $D_{k, t}$ im Querschnitt $k$ als Summe der Durchflußmengen aller Kanäle $y$ dieses Querschnitts angegeben werden: 
Es bedeuten

$$
\mathrm{D}_{\mathrm{k}, \mathrm{t}}=\underset{y=1}{\stackrel{\mathrm{N}}{\mathrm{N}} \mathrm{b}_{y, \mathrm{k}} \cdot \mathrm{H}_{y, \mathrm{k}, \mathrm{t}} \cdot \mathrm{U}_{y, \mathrm{k}, \mathrm{t}}}
$$

$\mathrm{b}_{y, \mathrm{k}} \quad$ : die Breite des Kanals $y$ im Querschnitt $\mathrm{k}$

$\mathrm{H}_{v, \mathbf{k}, \mathrm{t}}$ : die aktuelle Wassertiefe dieses Kanals

$\mathrm{U}_{v, \mathrm{k}, \mathrm{t}} \quad$ : die Geschwindigkeit im Kanal $v$ des Querschnittes $\mathrm{k}$.

Außerdem wird die örtliche Veränderung der Querschnitte in der Längsrichtung des Flusses berücksichtigt (RAmming 1962, 1968).

Abbildung 1 (Elbe, Normaltide 1951/1955) zeigt als Ergebnis, daß die Differenz zwischen berechneten und gemessenen Wasserständen maximal $3,5 \mathrm{~cm}$ beträgt. Bei

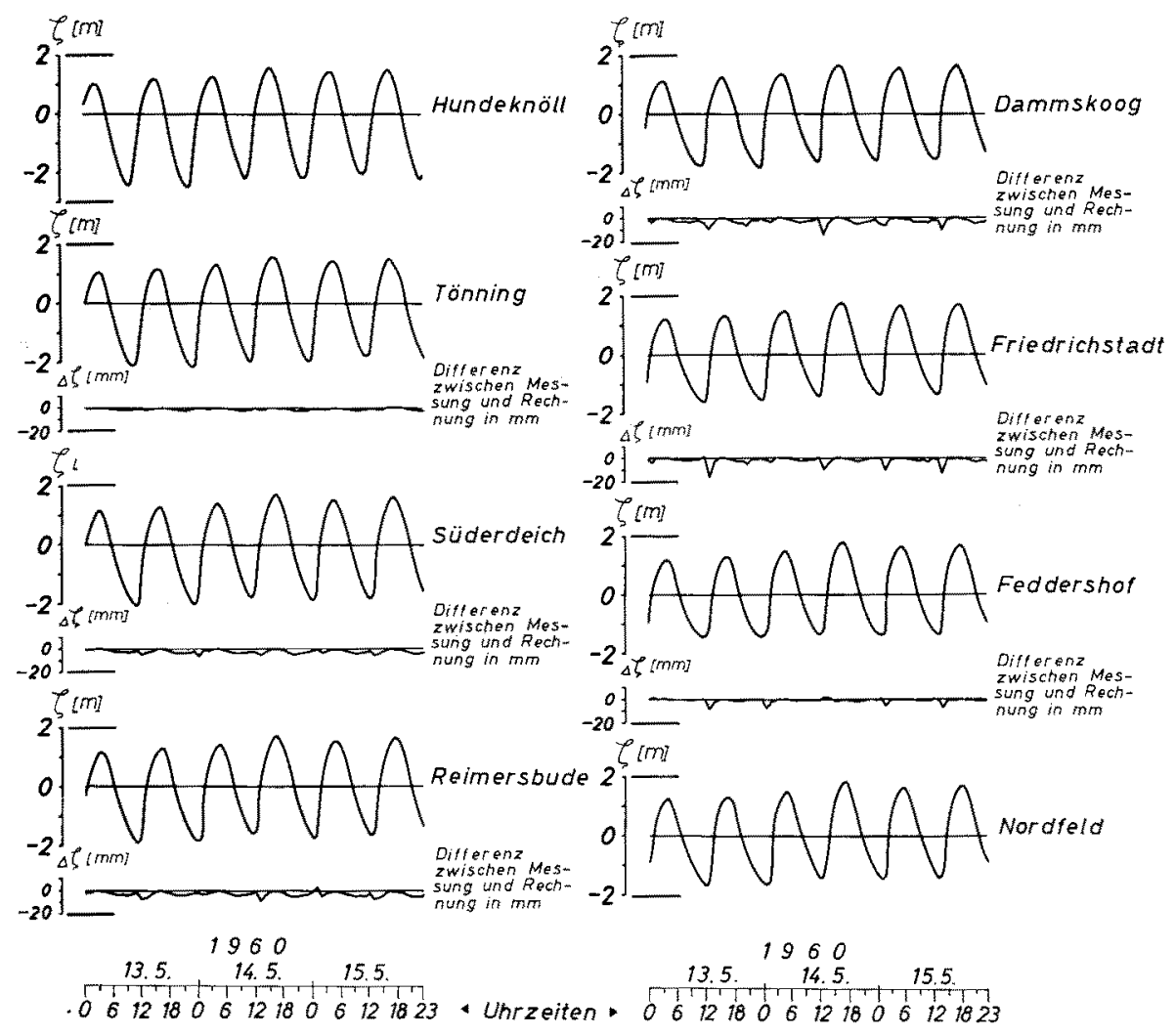

Abb. 2: Die Eider, Gezeit Mai 1960, Messung und Rechnung

Gezeitenberechnungen der Eider, ein Fluß, der eine sehr vielfältige Tiefen- und Querschnittsstruktur aufweist, sind, wie Abbildung 2 zeigt, über mehrere Perioden die Abweichungen zwischen Messung und Rechnung von der gleichen Größenordnung.

Die Ermittlung der Horizontalverteilung der Geschwindigkeit in einem Quer- 
schnitt schafft eine Möglichkeit, weiteren Einblick in die Bewegungsvorgänge zu gewinnen. Es ist bemerkenswert, daß in jedem Kanal der Vorgang des Kenterns des Stromes reproduziert wird, ebenso wie in der Natur, wo auch das Kentern nicht in allen Punkten eines Querschnittes zur gleichen Zeit erfolgt.

Abbildung 3 gibt den zeitlichen Verlauf der Geschwindigkeiten in einem Querschnitt der Eider wieder. Es ist deutlich zu erkennen, daß die Geschwindigkeiten in den Kanälen nicht zu gleicher Zeit Null sind. In dem hier gezeigten Beispiel wird der Querschnitt durch 10 Kanäle approximiert. Auf den nichtharmonischen Verlauf der Gezeiten- und Geschwindigkeitskurven sowie auf die Tatsache, daß nicht alle Kanäle durchgehend während einer Periode Wasser führen, sei besonders hingewiesen.
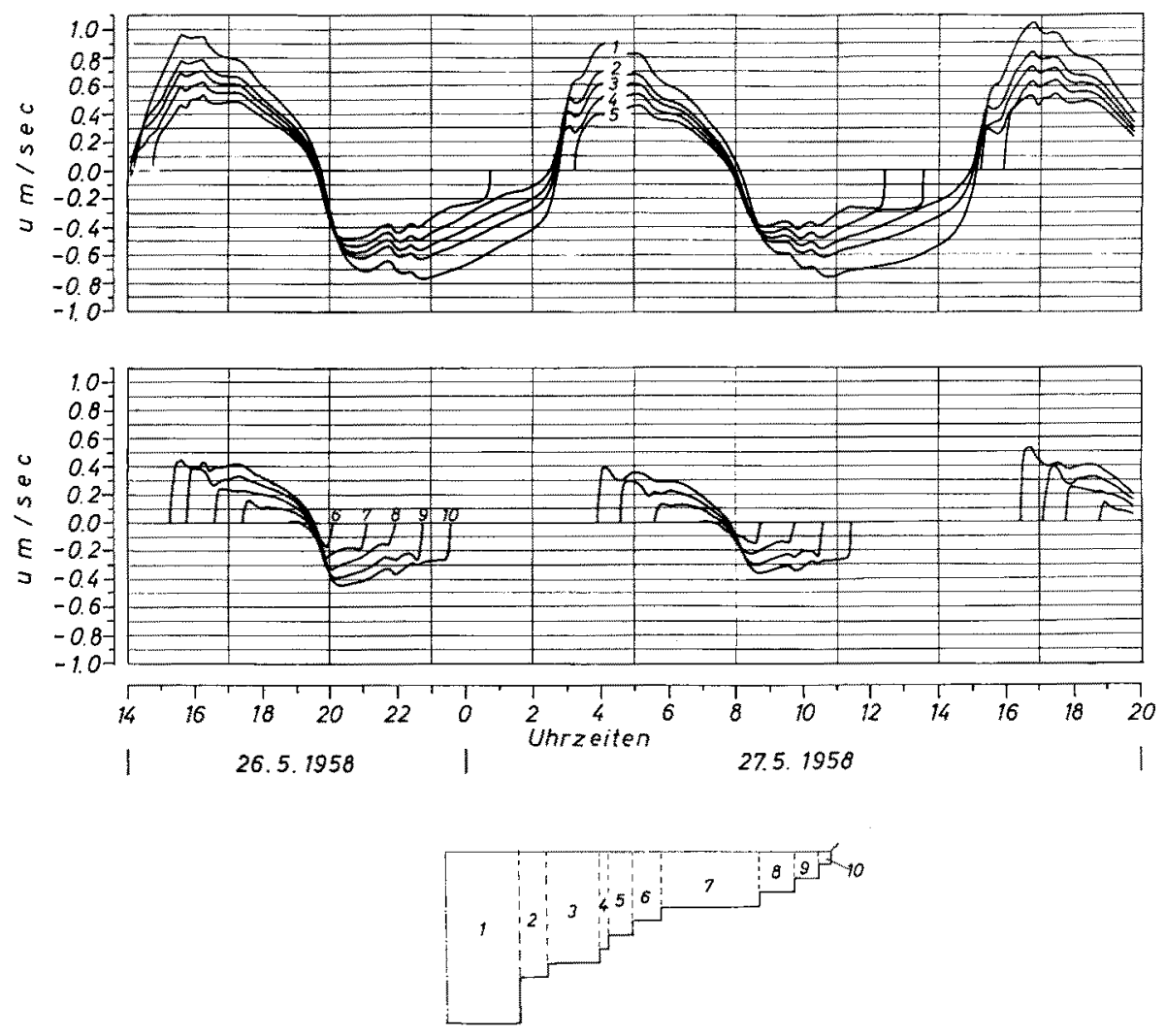

Abb. 3: Die Eider, Tide vom 26./27. Mai 1958. Berechnete Geschwindigkeiten in den 10 Lamellen des Querschnittes im Rechenpunkt 5 (km 93,45)

In Flachwassergebieten ist das Verhältnis der Amplitude zur Tiefe relativ groß, der Tidenhub kann sogar gleicher Größenordnung sein. Auf die nichtharmonische Gestalt der Wasserstands- und Geschwindigkeitskurven hat die Struktur der Morphologie besonderen Einfluß. Dieser Tatsache wird bei der numerischen Behandlung der hydrodynamischen Bewegungsgleichungen besonders Rechnung getragen. Für die Er- 
mittlung von Bewegungsvorgängen in Flachwassergebieten wird in der Bewegungsgleichung der quadratische Reibungsansatz $\mathbf{r}|\mathbf{u}| \mathbf{u}-\mathbf{r}$ ist eine konstante, dimensionslose Größe -, benutzt, und in beiden Gleichungen werden die nichtlinearen Terme, das heißt die zeitabhängige, aktuelle Wassertiefe, berücksichtigt.
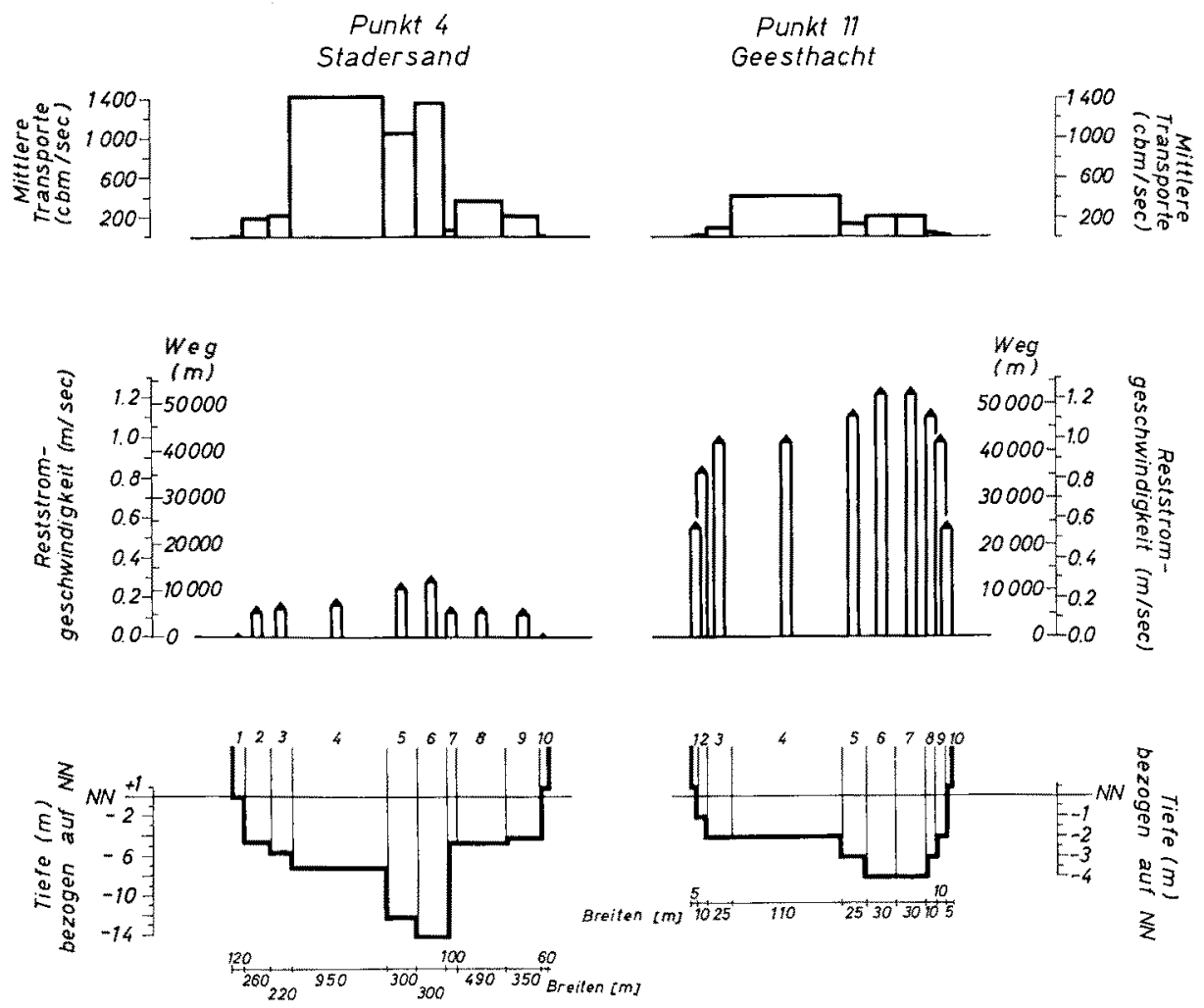

Abb. 4: Die Elbe, Normaltide 1951/1955, Reststromgeschwindigkeiten und mittlere Transporte

Wird nunmehr $\int_{0}^{T} \zeta_{k} d t$ und $\int_{0}^{T} u_{v}, k d t$ gebildet, so ergibt sich, daß der harmonischen Gezeitenbewegung, durch die das Wasser im Verlaufe einer Periode wieder an den Ausgangspunkt der Bewegung zurückkehrt, eine nichtharmonische Bewegung überlagert ist, die sich für die Horizontalbewegung als Reststrom und für die Vertikalbewegung als Abweichung vom mittleren Wasserstand eliminieren läßt und einen echten Massentransport verursacht, das heißt $\int_{0}^{T} \zeta_{\mathrm{k}} \mathrm{dt} \neq \mathrm{o}$ und $\int_{0}^{\mathrm{T}} \mathbf{u}_{v}, \mathrm{k} d \mathrm{~d} \neq \mathrm{o}$. Der Reststrom $\overrightarrow{\mathrm{u}}_{\mathrm{z}}, \mathrm{k}$ wird als über die Tiefe gemittelte, mittlere Geschwindigkeit einer Periode für jeden Kanal $y$ jedes Querschnittes $k$ eines Flusses ermittelt. Man erhält so eine Reststromverteilung für alle Querschnitte eines Flusses. Positives Vorzeichen der Rest- 
Transport von Verunreinigungen

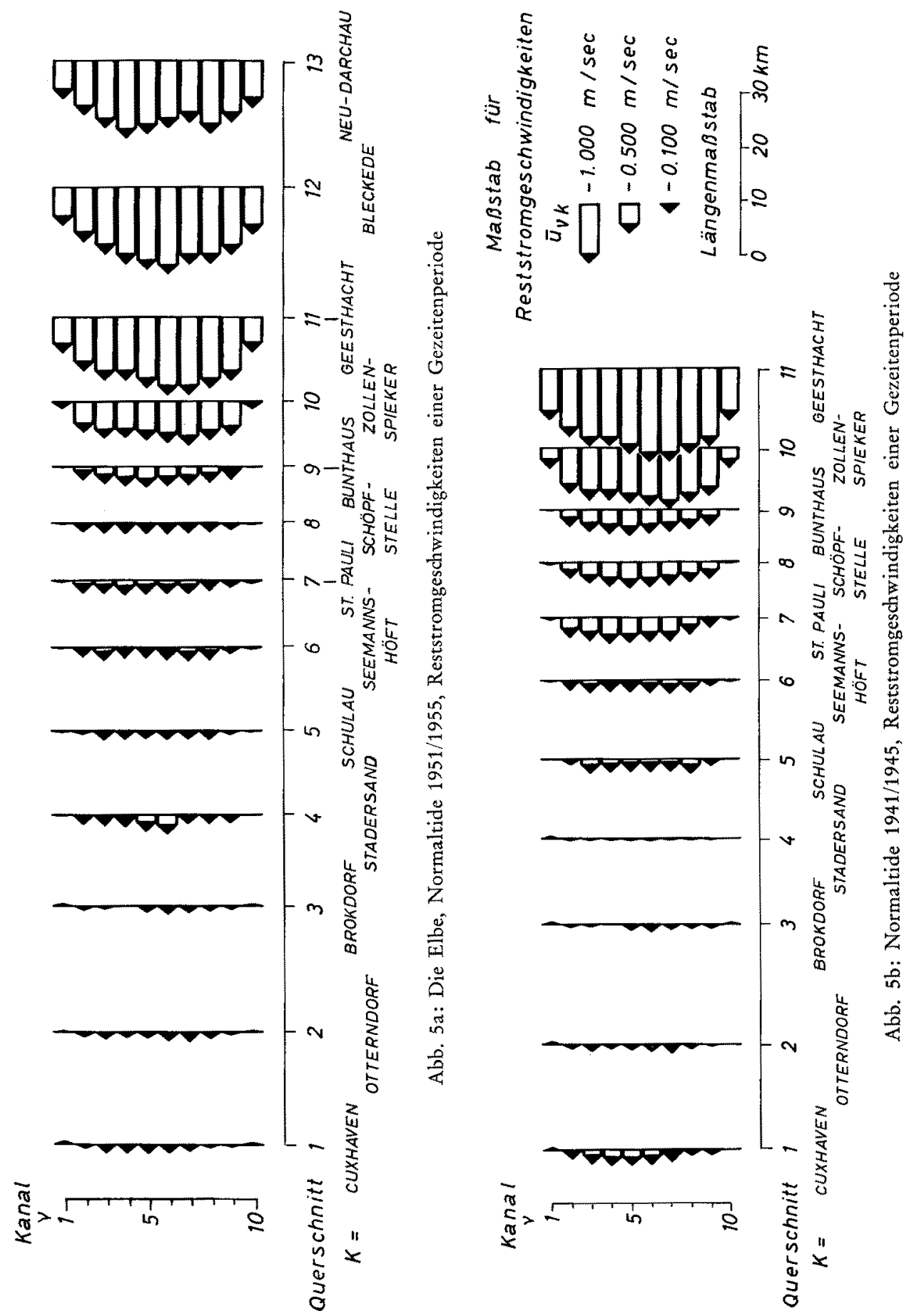


stromgeschwindigkeiten bedeutet flußaufwärts gerichtete und negatives Vorzeichen flußabwärts gerichtete Restströme.

Die so ermittelten Geschwindigkeiten $\overline{\mathbf{u}}_{v, \mathrm{k}}$ lassen sich für jeden Querschnitt als Funktion der Tiefe darstellen. Je größer die Tiefe $h_{v, k}$ eines Kanals, desto größer ist der Betrag der zugehörigen Geschwindigkeit $\overrightarrow{\mathrm{u}}_{v, \mathrm{k}}$. Es ist außerdem zu erwarten, daß in den Bereichen oberhalb der Gezeitengrenze, in denen die Geschwindigkeiten im wesentlichen von der zufließenden Oberwassermenge bestimmt werden, die Reststromgeschwindigkeit etwa der aktuellen Geschwindigkeit entsprechen wird. Es darf außerdem festgestellt werden, daß für die Untersuchung von Transporten und Verlagerungen nicht nur die maximalen Flut- und Ebbstromgeschwindigkeiten sowie die Flutund Ebbdauer, sondern besonders die Reststromgeschwindigkeiten zur Aussage herangezogen werden sollten.

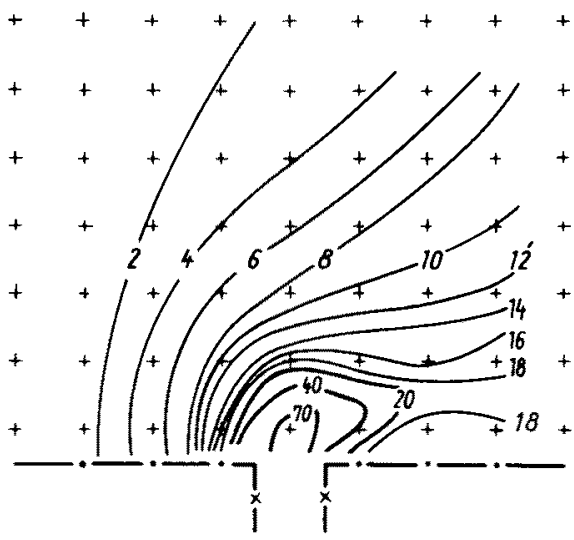

Abb. 6a

Abb. 6a: Strömungen, die durch den Ausfluß eines Stromes verursacht werden (Isogramm der Geschwindigkeiten, $\mathrm{cm} / \mathrm{sec}$ )

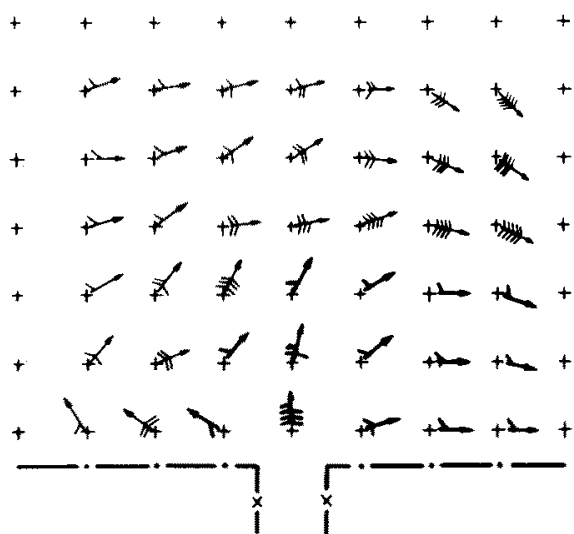

Abb. 6b

Abb. 6b: Strömungen, die durch den Ausfluß eines Stromes verursacht werden. Dünne Pfeile: $1-10 \mathrm{~cm} / \mathrm{sec}$; dicke Pfeile: $10-100$ $\mathrm{cm} / \mathrm{sec}$ (jeder Pfeil entspricht einer Einheit)

Abbildung 4 zeigt zwei Querschnitte eines Modellflusses, einen im Gezeitenbereich und einen im Oberwasserzuflußbereich. Es werden dargestellt: die für jeden Kanal $v$ ermittelten Reststromgeschwindigkeiten $\overline{\mathrm{u}}_{v, \mathrm{k}}$ und die aus der Breite $\mathrm{b}_{v, \mathrm{k}}$ der Wassertiefe $\mathrm{H}_{v, \mathrm{k}}$ und $\overrightarrow{\mathrm{u}}_{v, \mathrm{k}}$ berechneten Transporte.

Wird vorausgesetzt, daß keine Vermischung erfolgt und der Massentransport nur innerhalb der für das Modell ermittelten Kanalbreiten stattfindet, so läßt sich außerdem der resultierende Weg des Wassers innerhalb einer Gezeitenperiode, wie in der Abbildung 4 ebenfalls angegeben, aufzeigen.

In Abbildung $5 \mathrm{a}$ und $\mathrm{b}$ sind die Ergebnisse für zwei Normaltiden der Elbe dargestellt. Die für die Berechnung verwendeten Gezeitenkurven sind die über einen Zeitraum von jeweils 4 Jahren gemittelten, für jeden Pegel beobachteten zeitlichen Wasserstandswerte. Die Normaltidekurven wurden für diese Betrachtungen lediglich aus Vereinfachungsgründen gewählt. Es ist selbstverständlich möglich, wie sich auch aus dem bisher Gesagten ergibt, diese Untersuchungen für mehrere aufeinander folgende Perio- 
den einschließlich des Auftretens extrem hoher Wasserstände (Sturmfluten) durchzuführen.

Die ermittelten Reststromgeschwindigkeiten der beiden Normaltiden betragen in den Punkten 1 bis $9 \mathrm{zwischen} 1 \mathrm{~cm} / \mathrm{sec}$ und $35 \mathrm{~cm} / \mathrm{sec}$, während die Maximalwerte in

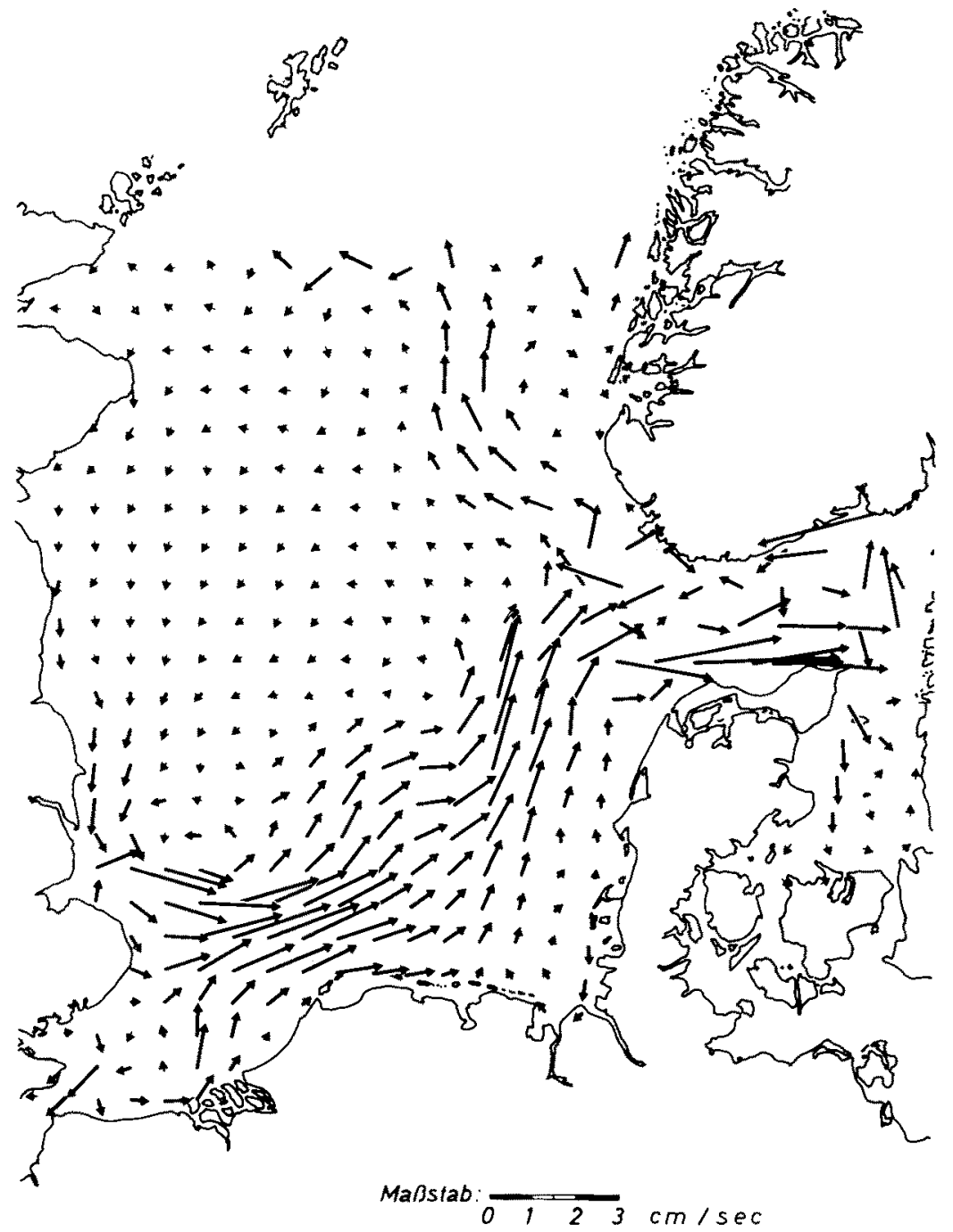

Abb. 7: Betrag und Richtung der Reststromgeschwindigkeit einer Periode der M2-Tide in der Nordsee

den Punkten 10 bis 13, als Folge des starken Oberwassereinflusses, auf etwa $130 \mathrm{~cm} / \mathrm{sec}$ steigen. Besonders zu beachten sind die positiven Reststromgeschwindigkeiten in den Uferkanälen der Punkte 1 bis 4; sie würden strömungsabhängiges Material stromaufwärts transportieren. 
Der unterschiedliche Gezeitenverlauf während der beiden zur Berechnung verwendeten Normaltiden hat andere Reststromgeschwindigkeiten zur Folge. Tägliche und andere zeitliche und örtliche Ungleichheiten der Gezeit, verschiedene Wirksamkeit der harmonischen Gezeitenanteile (Springtide, Nipptide u. a.), das Oberwasser und meteorologische Einflüsse verändern die Gestalt der Wasserstandskurven von Periode zu Periode, entsprechend werden die Reststromgeschwindigkeiten andere Werte annehmen. An dieser Stelle sei noch erwähnt, daß bei allen Rechnungen die ablenkende Kraft der Erdrotation berücksichtigt wurde. Es ist außerdem ohne Schwierigkeiten möglich, den Punktabstand zu verringern, um detaillierte Aussagen zu erhalten.

Es sollen nunmehr Folgerungen aus den bisher erzielten und dargestellten Ergebnissen für die Untersuchungen des Transportes von Verunreinigungen behandelt werden. Es wird vorausgesetzt, daß die verunreinigenden Stoffe im Wasser die Eigenschaften wie Wasser haben und sich in ihrer Bewegung strömungsabhängig verhalten. Eine chemische oder biologische Veränderung der Stoffe muß, soweit damit die Voraussetzung verletzt wird, ausgeschlossen werden. Verunreinigungen werden sich in tiefen Kanälen und großer, negativer Reststromgeschwindigkeit schneller zur Mündung bewegen als in flachen Kanälen. Sollen einem Fluß beispielsweise Industrieabwässer zugeführt werden, so sind die Auswahl des Ortes und die Wahl des Zeitpunktes - Ebbstrom - von Bedeutung, da sich das Material so kurze Zeit wie möglich im Fluß aufhalten soll. Anhand der ermittelten Reststromgeschwindigkeiten wurde zum Beispiel errechnet, daß ein Stoff, der die oben erwähnten Voraussetzungen erfüllt und in Hamburg der Elbe zugeführt wird, ca. 240 Stunden benötigt, um Cuxhaven zu erreichen.

Die bisherigen Arbeiten im Institut für Meereskunde der Universität Hamburg auf dem Gebiet des Transportes von Verunreinigungen haben sich vorerst lediglich auf die Untersuchung des Einflusses der Restströme beschränkt. Um einen tieferen Einblick in die Ausbreitungsvorgänge verunreinigten Wassers zu erhalten, scheint eine numerische Behandlung der Diffusionsgleichung durchaus sinnvoll, zumal damit auch die Ausbreitung von Stoffen unterschiedlicher Eigenschaften erfaßt werden kann. Erste Berechnungen wurden bereits durchgeführt.

Zum Abschluß sei noch auf zwei Ergebnisse hingewiesen: Abbildung 6 ( $a$ und $b$ ) zeigt die Strömungsverhältnisse vor einer Flußmündung. Küstenverlauf und Tiefenverteilung sind stark generalisiert. Der Einfluß der ablenkenden Kraft der Erdrotation auf die Geschwindigkeitsverteilung ist deutlich zu erkennen. In Abbildung 7 sind Betrag und Richtung der Reststromgeschwindigkeiten einer Periode der $\mathrm{M}_{2}$-Gezeit der Nordsee aufgetragen. Diese Darstellung vermittelt eine Vorstellung der Verlagerung der Wassermassen innerhalb einer $\mathrm{M}_{2}$-Periode. Ahnliche Untersuchungen über den Massentransport in der Nordsee als Folge dichte- und windbedingter Bewegungen wurden bereits durchgeführt.

\section{ZUSAMMENFASSUNG}

1. Die Anwendung des hydrodynamisch-numerischen Verfahrens hat bisher für verschiedene Meeresgebiete sowie für mehrere Gezeitenflüsse gute Ergebnisse gebracht. 
2. Durch Bildung von $\int_{0}^{T} \xi_{k} d t \neq 0$ und $\int_{0}^{T} u_{y, k} d t \neq 0$ wurde eine der harmonischen Gezeitenbewegung überlagerte, nichtharmonische Bewegung erfaßt.

3. Die auf diese Art ermittelten Restströme tragen zu einer echten Massenverlagerung und somit zum Transport von Verunreinigungen bei.

4. Wird nur die errechnete resultierende Transportgeschwindigkeit in den Querschnitten eines Flusses betrachtet, so können Aussagen über die Transportdauer gemacht werden.

5. Eine numerische Behandlung der Diffusionsgleichung erscheint durchaus sinnvoll, um die Ausbreitung von Verunreinigungen zu erfassen.

6. Mit Hilfe der vielseitigen Anwendungsmöglichkeiten des hydrodynamisch-numerischen Verfahrens können bemerkenswerte Beiträge zum Problem des Transportes und der Ausbreitung von Verunreinigungen in Flüssen und im Meere geleistet werden.

Ich danke Herm Prof. Dr. W. Hansen, Direktor des Instituts für Meereskunde der Universität Hamburg, für die anregenden und sehr eingehenden. Diskussionen über das Thema dieses Vortrages.

\section{ZITIERTE LITERATUR}

Hansen, W., 1955. Einfluß der Strömungen und Wasserstände in der Deutschen Bucht auf die Küstengestaltung. In: Tagungsbericht und wissenschaftiche Abhandlungen, Deutscher Geographentag, Hamburg 1955. Hrsg. von H. Wilhelmi \& K. H. SCHröder. Steiner, Wiesbaden, 346-350.

- 1966. The influence of coastal engineering structures on tides, currents and wind effects. Motion in the North Sea. Dock Harb. Auth. 46, 85-89.

- 1967. Die Reproduktion der Bewegungsvorgänge im Meere mit Hilfe hydrodynamischnumerischer Verfahren. Mitt. Inst. Meereskde Univ. Hamb, 5, 1-58.

Ramming, H. G., 1962. Gezeiten und Gezeitenströme in der Eider. In: Proceedings of the Symposium on mathematical-hydrodynamical methods of physical oceanography, Sept. 1961. Mitt. Inst. Meereskde Univ. Hamb. 1, 233-237.

- 1968. Shallow water tides. Mitt. Inst. Meereskde Univ. Hamb. 10, 64-68.

\section{Diskussion im Anscbluß an den Vortrag RAMMING}

CASPERS: Wir beobachten in Süßwasserbereich der Elbe Schalen mariner Organismen (Benthosformen wie Foraminiferen, Plankter). Werden diese im Wasser (Restwasser?) elbaufwärts transportiert oder ist eine sukzessive Deponierung am Boden exforderlich?

Ramming: Ein stromaufwärts gerichteter Transport kann möglich sein. Ob dieser Transport bis Hamburg im Verlaufe mehrerer aufeinander folgender Gezeitenperioden stattfindet, vermag ich nicht zu sagen. Ich darf in diesem Zusammenhang darauf hinweisen, daß zur Zeit hydrodynamisch-numerische Untersuchungen über die Vertikalverteilung der Geschwindigkeit erfolgen, so daß dann eine eingehendere Beantwortung möglich sein wird.

CAspers: Ist bei thren Berechnungen die Rolle der Wattenzonen des Stromes zu kalkulieren?

Ramming: Die Wattenzonen gehen in die Rechnung ein. Ich verweise auf die positiv gerichteten Reststromgeschwindigkeiten zwischen Cuxhaven und Glückstadt. 